\title{
Immunologic correlates of sexual dimorphism in human and simian immunodeficiency virus vaccine efficacy
}

\author{
Marjorie Robert-Guroff*,1 \\ ${ }^{1}$ National Cancer Institute, Vaccine Branch, 41 Medlars Drive, Building 41, Room D804, Bethesda, MD 20852-5065, USA \\ *Author for correspondence: Tel.: 240760 6616; Fax: 240541 4454; guroffm@mail.nih.gov
}

"The effort needed to make sure that a future vaccine is protective for all vaccinees has become more difficult. Design and testing of vaccines in females is more complex than in males, but the effort is critical, as approximately $50 \%$ of HIV infections worldwide occur in women."

First draft submitted: 25 October 2018; Accepted for publication: 12 November 2018; Published online: 3 December 2018

Keywords: HIV and SIV vaccines • innate and adaptive immunity • sex bias

Sex differences in susceptibility to various viral infections and subsequent pathogenesis are well-known [1]. These differences extend to HIV infection and disease development, where initially women exhibit lower viral loads and higher CD4 T-cell counts, but paradoxically exhibit more rapid disease progression [1,2]. This phenomenon has been attributed to elevated immune responses in women following viral infection, including pathogen recognition by pattern recognition receptors, induction of innate and adaptive immune responses and production of inflammatory cytokines. These responses may fail to return to baseline after viral clearance, thus contributing to pathogenesis [1]. The sex bias in general is associated with differences in both innate and adaptive immunity [3], attributed to multiple factors including sex hormones, $x$-linked genes, and the microbiome [2]. As the latter plays an important role, it is not surprising that the sex bias particularly effects mucosal immunity [4].

In addition to their response to viral infections, females, both children and adults, exhibit greater immune responses to vaccines including those against influenza, hepatitis $\mathrm{B}$, herpes virus, yellow fever virus, rabies and small pox. Only after the age of 65 do males develop greater immunity than females to tetanus/diphtheria/pertussis and pneumococcal vaccines [5]. In spite of these distinct differences, few studies have reported any influence of sex on vaccine-induced protective efficacy. A vaccine against HSV-2 was reported to induce significant protective efficacy in females but not males [6]. Of interest to this commentary, in the RV144 HIV vaccine trial in Thailand, greater efficacy was observed in women compared with men, although the difference was nonsignificant [7]. If sex plays a meaningful role in HIV vaccine efficacy, it would be important to identify immune correlates associated with the protection in order to appropriately design effective vaccine regimens for both men and women.

Rhesus macaques have become the nonhuman primate model (NHP) of choice for preclinical evaluation of HIV vaccine strategies. Although not susceptible to infection by HIV, simian immunodeficiency virus (SIV) infection of macaques adequately mimics HIV transmission and disease progression in humans. Additionally, SIV-HIV chimeric viruses can be used for evaluation of vaccines specifically targeting the HIV envelope. Using the rhesusSIV model, we uncovered a sex bias in vaccine-induced protective efficacy, whereby female but not male macaques exhibited significantly delayed SIV acquisition following low-dose SIV mac251 intrarectal challenge [8]. The delayed acquisition correlated significantly with local mucosal B cell immunity, including SIV envelope (Env)-specific rectal immunoglobulin A (IgA), rectal SIV Env-specific memory B cells and total rectal plasma cells. Subsequent follow-up studies identified additional immune responses correlated with protection. Although the male macaques developed higher serum antibody responses, results suggested that the female macaques developed better antibody quality with enhanced functionality, as their Env-specific IgG3 antibodies but not those of the males correlated directly with antibody-dependent cellular cytotoxicity (ADCC) and antibody-dependent cellular phagocytosis (ADCP) [9]. The female IgG3 antibodies also correlated with decreased peak viremia following infection, not seen in the male 
macaques. A possible mechanism contributing to this improved antibody quality, was suggested by the finding that the vaccinated female macaques developed greater frequencies of SIV Env-specific T-follicular helper cells postvaccination. These were significantly correlated with serum binding antibody titers, the frequency of rectal plasma blasts and ADCC killing activity [10]. However, along with enhanced antibody activity, the male macaques also developed greater frequencies of B regulatory cells, able to suppress T-cell proliferation and directly correlated with peak viremia [9]. This suggested a potential mechanism facilitating SIV acquisition in the males, perhaps enhancing the sex differential.

While the Tuero and follow-up studies identified numerous immune responses in females potentially benefitting their vaccine outcome, a recent preclinical study showed that females don't always have the upper hand. As reported at the 36th Annual Symposium on Nonhuman Primate Models for AIDS, Seattle, WA, USA, male macaques but not females exhibited protection against a $\mathrm{SHIV}_{1157 \mathrm{ipd} 3 \mathrm{~N} 4}$ challenge following priming and boosting with a modified vaccinia Ankara (MVA)/gp145 ALFA-adjuvanted (Army Liposome Formulation with aluminum hydroxide [11]) vaccine regimen (Diane Bolton, personal communication). A decreased per-exposure risk of $92 \%(p=0.003$ ) was seen in contrast to a similar regimen adjuvanted with alum hydroxide in which no protection was observed in either males or females. The delayed acquisition in vaccinated males was associated with ADCP activity; further analyses are ongoing to identify other immune correlates which may have affected the outcome. The impact of the alum versus ALFA adjuvant [11] on induced immune responses and protective outcome is also a subject of intense ongoing investigation.

In addition to the importance of identifying factors associated with the vaccine-mediated delayed SHIV acquisition of males in the Bolton study, the distinctly different outcome compared with the Tuero study suggests a need to examine differences in overall vaccine regimens. Tuero et al. utilized mucosal priming with replication-competent Adenovirus type 5 host range mutant (Ad5hr)-SIV recombinants, followed by boosting systemically with either monomeric gp120 or oligomeric gp140 in MF59 adjuvant. In contrast, Bolton and colleagues utilized systemic priming with recombinant MVA followed by boosting systemically with liposomal-adjuvanted gp145. Certainly, there could be differences introduced due to the different adjuvants, as already highlighted in the Bolton study. However, could the differential delay in acquisition in females and males seen in the two studies be in part due to the vaccination route? A study in mice although focused on induction of cellular immunity explored immunization with an HIV Env peptide, a DNA plasmid and a MVA recombinant. Optimal immune responses in the spleen, reproductive tract and colon of female mice were achieved by mucosal nasal peptide priming followed by systemic (intradermal) MVA boosting, although this regimen was poorly immunogenic at comparable sites in males [12]. In contrast, an intradermal MVA prime followed by an intradermal MVA boost elicited the best immune responses in the spleen, reproductive tract and lungs of male mice. Thus, different immunization routes were needed to induce mucosal immune responses in the males and females. Steroid hormones are known to modulate the microbiome [13], which in turn helps shape mucosal immune responses [14]. It would be remarkable if equivalent HIV protective efficacy could be induced in men and women simply by altering immunization routes.

Antibodies, both neutralizing and non-neutralizing have become key targets for HIV vaccine design. As seen in both studies discussed here, vaccine-induced antibodies with functional activity appear to be contributing factors in the sex biases in vaccine outcome. It is known that glycosylation differences in the antibody Fc region modulate antibody-dependent effector functions such as ADCC and ADCP activities [15]; they also impact HIV control [16]. Further, Fc glycosylation differences are known to exist between males and females [17]. We pursued the potential impact of Fc glycosylation on the vaccine-induced sex bias while investigating another antibody effector function, antibody-dependent complement mediated lysis (ADCML) of SIV and SIV-infected cells using sera from the same Tuero study [18]. Sera of all vaccinated macaques induced ADCML of SIV and SIV-infected cells similarly, regardless of sex. However, sex differences were seen in vaccine-induced antibody characteristics and functions where certain Ig subtypes correlated with SIV lysis in females whereas others were associated with SIV-infected cell lysis in males. Importantly, using a systems serology approach, we found that females had increased Env-specific IgG G2S1 glycoforms which correlated with ADCML while males had greater levels of Env-specific IgG G1'/G0FB and G1'FB/G2 glycoforms that correlated with ADCML, substantiating the impact of Fc glycosylation on antibody differences between the sexes and identifying a mechanism by which other sex differences in humoral responses may arise.

Many parameters other than what have been documented here could impact vaccine-induced protection. For example, FcR polymorphisms modulate binding affinity and affect antibody-dependent functions [19]. Could these differ between males and females? Regulatory $\mathrm{T}$ cells contribute to the sex difference in the prevalence 
of autoimmune diseases [20]. Could this difference impact vaccine efficacy? Characteristics of antibodies elicited by vaccines may differ in many properties such as epitope specificities, potency, avidity and the potential for antibody-dependent enhancement. How will these properties affect vaccine outcomes between the sexes?

The realization that sex differences occur in HIV/SIV vaccine efficacy, although not seen in all studies, impacts both preclinical and clinical vaccine trials and will greatly increase the complexity of vaccine design and evaluation. It will require balancing of the sexes in both NHP and human studies and require study of the microbiome and indepth examination of immune responses beyond mere quantitation of functional activities. Effects of different adjuvants and immunization routes will need to be probed, and levels and properties of innate and adaptive immune cells, including those that regulate immune responses and those that perform effector functions, will need to be investigated. Considering hormonal effects, the extent to which sex differences are preserved or altered in children versus adults will need to be addressed as HIV pediatric vaccines move into clinical trials. Development of an HIV vaccine is a daunting task. The effort needed to make sure that a future vaccine is protective for all vaccinees has become more difficult. Design and testing of vaccines in females is more complex than in males, but the effort is critical, as approximately $50 \%$ of HIV infections worldwide occur in women.

\section{Acknowledgments}

This work was supported by the Intramural Research Program of the National Institutes of Health, National Cancer Institute.

\section{Financial \& competing interests disclosure}

The author has no relevant affiliations or financial involvement with any organization or entity with a financial interest in or financial conflict with the subject matter or materials discussed in the manuscript. This includes employment, consultancies, honoraria, stock ownership or options, expert testimony, grants or patents received or pending, or royalties.

No writing assistance was utilized in the production of this manuscript.

\section{References}

1 Klein SL. Sex influences immune responses to viruses, and efficacy of prophylaxis and treatments for viral diseases. Bioessays 34(12), 1050-1059 (2012).

2 Rechtien A, Altfeld M. Sexual dimorphism in HIV-1 infection. Semin. Immunopathol. doi:10.1007/s00281-018-0704-y (2018) (Epub ahead of print).

3 Klein SL, Flanagan KL. Sex differences in immune responses. Nat. Rev. Immunol. 16(10), 626-638 (2016).

4 Sankaran-Walters S, Macal M, Grishina I et al. Sex differences matter in the gut: effect on mucosal immune activation and inflammation. Biol. Sex Differ. 4(1), 1-12 (2013).

5 Flanagan KL, Fink AL, Plebanski M, Klein SL. Sex and gender differences in the outcomes of vaccination over the life course. Annu. Rev. Cell Dev. Biol. 33, 577-599 (2017).

6 Stanberry LR, Spruance SL, Cunningham AL et al. Glycoprotein-D-adjuvant vaccine to prevent genital herpes. N. Engl. J. Med. 347(21), 1652-1661 (2002).

7 Rerks-Ngarm S, Pitisuttithum P, Nitayaphan S et al. Vaccination with ALVAC and AIDSVAX to prevent HIV-1 infection in Thailand. N. Engl. J. Med. 361(23), 2209-2220 (2009).

8 Tuero I, Mohanram V, Musich T et al. Mucosal B cells are associated with delayed SIV acquisition in vaccinated female but not male rhesus macaques following SIV $_{\text {mac251 }}$ rectal challenge. PLoS Pathog. 11(8), e1005101 (2015).

9 Mohanram V, Demberg T, Musich T et al. B cell responses associated with vaccine-induced delayed SIV $_{\text {mac251 acquisition in female }}$ rhesus macaques. J. Immunol. 197(6), 2316-2324 (2016).

10 Vargas-Inchaustegui DA, Demers A, Shaw JA et al. Vaccine-induction of LN-resident SIV Env-specific $\mathrm{T}_{\mathrm{FH}}$ cells in rhesus macaques. J. Immunol. 196(4), 1700-1710 (2016).

11 Beck Z, Torres OB, Matyas GR, Lanar DE, Alving CR. Immune response to antigen adsorbed to aluminum hydroxide particles: effects of co-adsorption of ALF or ALFQ adjuvant to the aluminum-antigen complex. J. Control. Rel. 275, 12-19 (2018).

12 Peacock JW, Nordone SK, Jackson SS et al. Gender differences in human immunodeficiency virus type 1-specific CD8 responses in the reproductive tract and colon following nasal peptide priming and modified vaccinia virus Ankara boosting. J. Virol. 78(23), 13163-13172 (2004).

13 Markle JG, Fish EN. SeXX matters in immunity. Trends Immunol. 35(3), 97-104 (2014).

14 Honda K, Littman DR. The microbiome in infectious disease and inflammation. Annu. Rev. Immunol. 30, 759-795 (2012).

15 Chung AW, Crispin M, Pritchard L et al. Identification of antibody glycosylation structures that predict monoclonal antibody Fc-effector function. AIDS 28(17), 2523-2530 (2014). 


\section{Editorial Robert-Guroff}

16 Ackerman ME, Crispin M, Yu X et al. Natural variation in Fc glycosylation of HIV-specific antibodies impacts antiviral activity. J. Clin. Invest. 123(5), 2183-2192 (2013).

17 Chen G, Wang Y, Qiu L et al. Human IgG Fc-glycosylation profiling reveals associations with age, sex, female sex hormones and thyroid cancer. J. Proteomics 75(10), 2824-2834 (2012).

18 Miller-Novak LK, Das J, Musich TA et al. Analysis of complement-mediated lysis of SIV and SIV-infected cells reveals sex differences in vaccine-induced immune responses in rhesus macaques. J. Virol. 92(19), e00721-e007218 (2018).

19 Nimmerjahn F, Ravetch JV. Fcgamma receptors as regulators of immune responses. Nat. Rev. Immunol. 8(1), 34-47 (2008).

20 Afshan G, Afzal N, Qureshi S. CD4+CD25(hi) regulatory T cells in healthy males and females mediate gender difference in the prevalence of autoimmune diseases. Clin. Lab. 58(5-6), 567-571 (2012). 